\title{
Magnetic Activity Following Re-Accretion on to Galaxies
}

\author{
H. Nishikori \\ Graduate School of Science and Technology, Chiba University, 1-33, \\ Yayoi-cho, Inage-ku, Chiba 263-8522, Japan \\ M. Machida
}

National Astronomical Observatory of Japan, 2-21-1, Osawa, Mitaka, Tokyo 181-8588, Japan

R. Matsumoto

Department of Physics, Faculty of Science, Chiba University, 1-33, Yayoi-cho, Inage-ku, Chiba 263-8522, Japan

\begin{abstract}
We carried out global three-dimensional magnetohydrodynamical (MHD) simulations of galactic gaseous disks re-accreting intergalactic plasma. As the initial condition, we assume that a rotating slender torus is formed at $10 \mathrm{kpc}$ from the galactic center. We assume a gravitational potential generated by bulge stars, disk stars and dark matters. Numerical results indicate that magnetorotational instability (MRI) growing in the torus amplifies magnetic fields and generates turbulence. The Maxwell stress enhanced by turbulent magnetic fields drives mass accretion of the disk gas. The amplification of magnetic fields in the accreting gas disk drives magnetic activities such as flares and plasma heating due to magnetic reconnection. The magnetic activity is maintained for time scales longer than the accretion time scale, typically $5 \mathrm{Gyr}$.
\end{abstract}

When a disk galaxy captures an intergalactic dense blob, such as a dwarf galaxy, it will form a gas torus rotating in the galactic gravitational potential. We carried out global three-dimensional MHD simulations of the evolution of the gas torus.

As the initial distribution of gas density, we assume that a rotating torus is embedded in an isothermal,non-rotating hot halo. As the gravitational potential, we adopted an axisymmetric model of our galaxy given by Miyamoto et al. (1980), which includes the contribution from dark matter. The initial magnetic field is assumed to be weak $\left(\beta=P_{g a s} / P_{m a g}=100\right)$ and toroidal. We solved the resistive MHD equations in a cylindrical coordinate system $(\varpi, \varphi, z)$ by using a Modified Lax-Wendroff scheme with artificial viscosity. The number of mesh points is $\left(N_{\varpi}, N_{\varphi}, N_{z}\right)=(250,64,319)$. We adopted absorbing inner boundary condition at $\varpi=0.6 \mathrm{kpc}$. Figure 1 (left) shows the initial condition.

As MRI grows in the torus, magnetic turbulence is generated. The Maxwell stress exerted by the turbulent magnetic fields drives accretion of the disk material. As the matter accretes to the central region, a flat gas disk is formed (Figure 1, right). The accretion timescale defined by the time when half of the 

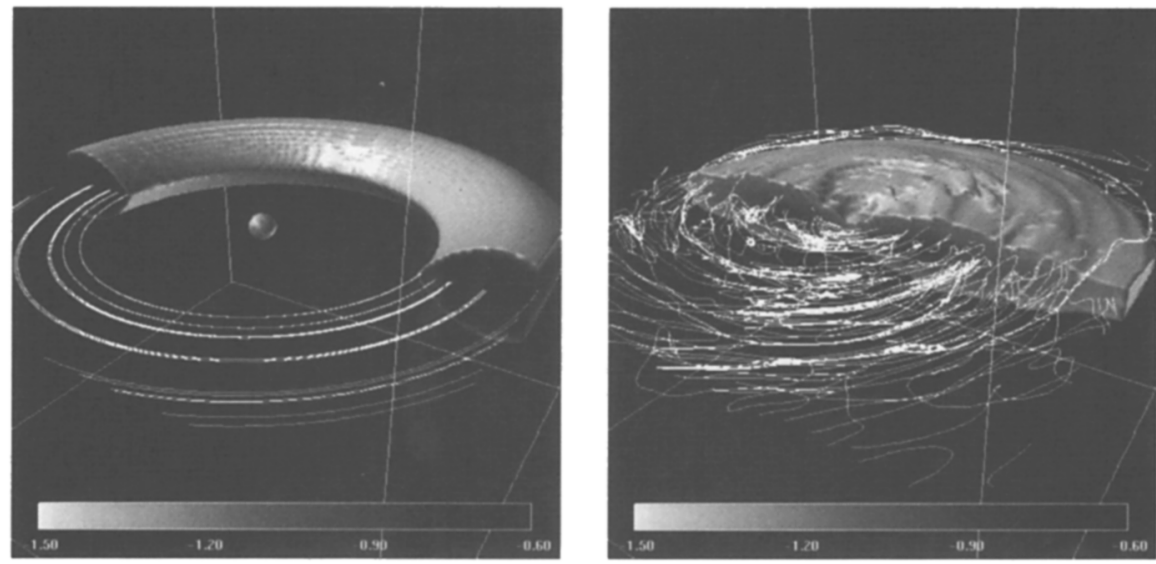

Figure 1. Left: Initial model of the simulation. The gray scale shows the density distribution. Azimuthal magnetic fields are assumed to be in the torus. The box size is $24 \mathrm{kpc} \times 24 \mathrm{kpc} \times 24 \mathrm{kpc}$. Right: Three dimensional view of simulation results after $5.3 \mathrm{Gyr}$.

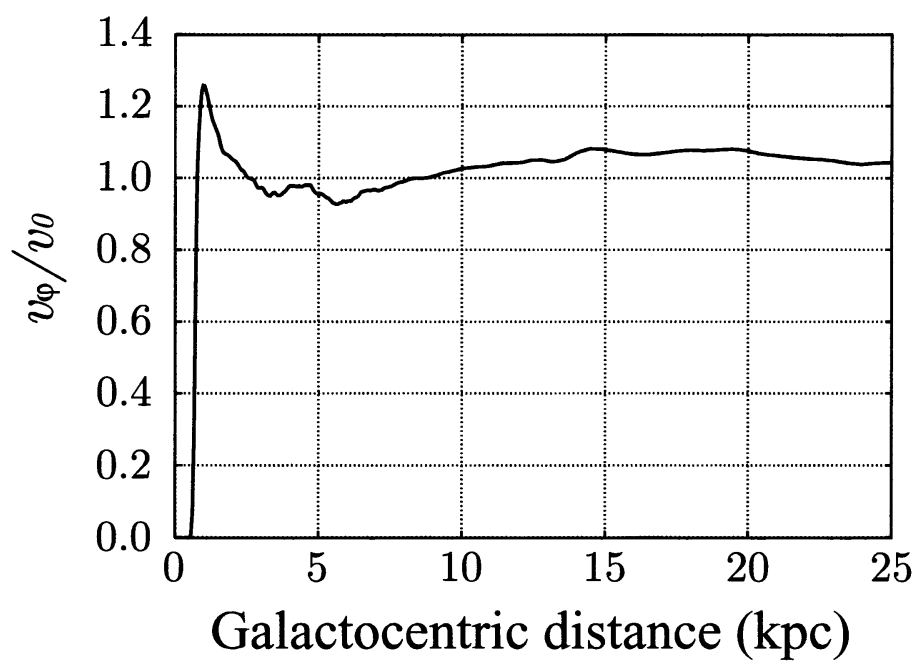

Figure 2. The azimuthal velocity averaged in $0 \mathrm{kpc}<z<0.3 \mathrm{kpc}, 0<$ $\varphi<2 \pi$ after $5.3 \mathrm{Gyr} ; v_{0} \sim 220 \mathrm{~km} \mathrm{~s}^{-1}$.

initial mass of the torus accretes inside the inner boundary is 3.2Gyr. Turbulent magnetic fields create numerous current sheets inside the disk. Magnetic reconnection taking place in the current sheet can heat up the interstellar matter. The azimuthal velocity averaged in $0 \mathrm{kpc}<z<0.3 \mathrm{kpc}$ and $0<\varphi<2 \pi$ has the profile similar to that observed in our Galaxy (Figure 2). 
Acknowledgments. Numerical computations were carried out on VPP5000 at the Astronomical Data Analysis Center of the National Astronomical Observatory, Japan (yhn28b, P.I. H. Nishikori).

\section{References}

Miyamoto, M., Satoh, C., \& Ohashi, M. 1980 A\&A, 90, 215 